Marquette University

e-Publications@Marquette

School of Dentistry Faculty Research and

Publications

Dentistry, School of

$11-1-2008$

\title{
Altering Occlusal Vertical Dimension Provisionally with Base Metal Onlays: A Clinical Report
}

Soni Prasad

Marquette University, soni.prasad@marquette.edu

Jennifer Kuracina

State University of New York at Buffalo

Edward A. Monaco, Jr.

State University of New York at Buffalo

Accepted version. The Journal of Prosthetic Dentistry, Vol. 100, No. 5 (November 2008): 338-342.

DOI. (C) 2008 Elsevier B.V. Used with permission.

Soni Prasad was affiliated with the State University of New York at Buffalo at the time of publication. 


\section{Marquette University}

\section{e-Publications@Marquette}

\section{Dentistry Faculty Research and Publications/School of Dentistry}

This paper is NOT THE PUBLISHED VERSION; but the author's final, peer-reviewed manuscript. The published version may be accessed by following the link in the citation below.

The Journal of Prosthetic Dentistry, Vol. 100, No. 5 (November, 2008): 338-342. DOI. This article is (C) Elsevier and permission has been granted for this version to appear in e-Publications@Marquette. Elsevier does not grant permission for this article to be further copied/distributed or hosted elsewhere without the express permission from Elsevier.

\section{Altering Occlusal Vertical Dimension Provisionally with Base Metal Onlays: A Clinical Report}

\section{Soni Prasad}

Implant Fellow, Postgraduate Prosthodontics, Department of Restorative Dentistry, School of Dental Medicine, State University of New York at Buffalo, Buffalo, NY

Jennifer Kuracina

Clinical Assistant Professor, Postgraduate Prosthodontics, Department of Restorative Dentistry, School of Dental Medicine, State University of New York at Buffalo, Buffalo, NY

\section{Edward A. Monaco Jr}

Clinical Assistant Professor and Director, Postgraduate Prosthodontics, Department of Restorative Dentistry, School of Dental Medicine, State University of New York at Buffalo, Buffalo, NY

This article presents a method for altering occlusal vertical dimension (OVD) to restore dentitions with limited restorative space due to loss of tooth structure. A provisional increase in OVD is achieved using predominately base metal onlays, which offer advantages over conventional techniques. The onlays are resistant to wear, may be bonded well to nonrestored and restored tooth surfaces with resin cements, and provide a fairly reversible method of increasing OVD. A patient situation is presented which demonstrates the use of provisional base metal onlays in complete mouth rehabilitation. (J Prosthet Dent 2008;100:338-342) 
Patients often seek rehabilitative treatment for severely worn dentition. $\frac{1}{}$ The etiology of noncarious tooth wear due to attrition, abrasion, and erosion is multifactorial.. To treat the worn dentition, it is important to identify and eliminate and/or control the factors that contribute to excessive wear. This article primarily pertains to tooth wear related to diet and parafunction.

Restoration of a worn dentition is challenging when a lack of restorative space exists. Occlusal vertical dimension is, by definition, the distance measured between 2 points when teeth are in contact. -3 Loss of tooth structure does not necessarily mean loss of OVD. $\frac{4}{5}, \underline{\text { It }}$ may be difficult to determine if OVD has been lost. Therefore, rather than focusing on determining whether OVD is lost, another solution may be to evaluate whether the proposed alteration in occlusal vertical dimension is restoratively acceptable. The treatment of a patient demonstrating excessive tooth wear is described. Although OVD appeared to have been maintained, on diagnostic evaluation based on a diagnostic waxing, there was insufficient posterior tooth structure. As a result, an alteration in the OVD was required to provide adequate tooth structure for rehabilitation of the dentition.

There are many methods cited in the literature for evaluating OVD. $\underline{6}$ Assessing the rest vertical dimension is one way to clinically appraise the OVD. The flaw with this technique is that interocclusal distance may be variable. $\underline{\underline{n}}$ Evaluating posterior speaking space, $, \underline{\underline{g}}, \underline{\underline{9}}, \underline{10} \mathrm{facial}$ soft tissue contours, $\underline{11}$ and cephalometrics are other methods of assessing OVD. Despite these techniques, there is no absolute method to determine an acceptable OVD. However, speech and function can be used to clinically evaluate an acceptable OVD. .9

Clinicians may decide to increase OVD based on the amount of interocclusal space required to restore the dentition to proper esthetics, form, and function. If an increase is indicated and performed, it should be followed up for several months. ${ }^{6}$ Conventionally, the increase in OVD is achieved either with a removable acrylic resin occlusal splint or with the use of provisional restorations, for example, direct bonded composite resin or provisional fixed restorations. $\underline{\underline{6}}$ Compliance may be an issue with removable occlusal splints, and speech interference may also be a common problem. A conventional fixed provisional restoration, even when heat polymerized, may wear during the evaluation period or over the span of treatment, and, therefore, may make long-term management of OVD difficult. Loss of cement seal and irreversible tooth preparation are additional problems associated with fixed provisional restorations.

Base metal (BM) onlays provide a fairly reversible approach. The onlays can be bonded to unprepared or restored tooth surfaces so that an increase in OVD can be evaluated. Due to the wear resistance of the base metal onlays, the altered OVD is maintained throughout the evaluation period, even permitting complete mouth rehabilitation to be accomplished in segments over a long period of time.

The advantages of using BM onlays to increase occlusal vertical dimension include: (1) no preparation is required prior to evaluation of increased OVD; (2) patient compliance is not an issue; (3) BM onlays are strong and wear resistant; (4) they bond well to a variety of tooth and restorative surfaces with resin cements $\underline{\underline{12}}$; (5) BM onlays allow for occlusal adjustments when needed; (6) BMs can be removed when the patient is ready for the fabrication of definitive 
restorations at the newly established OVD; (7) the potential for fracture and loss of cement seal of provisional restorations is decreased with BMs; and (8) BM onlays allow for a segmental restorative treatment approach for situations requiring complete mouth restorations.

The disadvantages of using base metal onlays include: (1) modest increases in laboratory procedures and cost; (2) adjustment is typically more difficult due to the hardness of BMs; (3) they are unesthetic and may not be suited for all treatment scenarios; (4) removal of onlays may be difficult; (5) judicious techniques are required if premature removal of onlays becomes necessary; and (6) they are not recommended for all OVD situations.

This article demonstrates a method of complete mouth restoration using BM onlays to increase OVD in a situation with limited restorative space due to loss of tooth structure.

\section{Clinical report}

A 73-year-old white man presented with a severely worn dentition and desired to have his teeth restored for improved esthetics and function. An anterior open articulation was observed, as the mandibular central incisors were worn to the gingival margin. Numerous existing restorations were present. Significant generalized wear and evidence of parafunction were observed (Fig. 1, Fig. 2, Fig. 3). Treatment plan options were presented to the patient. A complete mouth rehabilitation with metal ceramic restorations was suggested. The patient was given the following options: restoration of the edentulous left posterior region with implants, a removable partial denture, or no tooth replacement, leaving the left side with premolar occlusion. The patient decided to have implants placed. Although the patient was eager to restore his dentition for esthetics and function, time commitments and financial concerns prevented him from seeking complete mouth rehabilitation. Rehabilitation using a segmental approach was suggested, which gave him financial flexibility and a comfortable occlusion during the transitional phase.

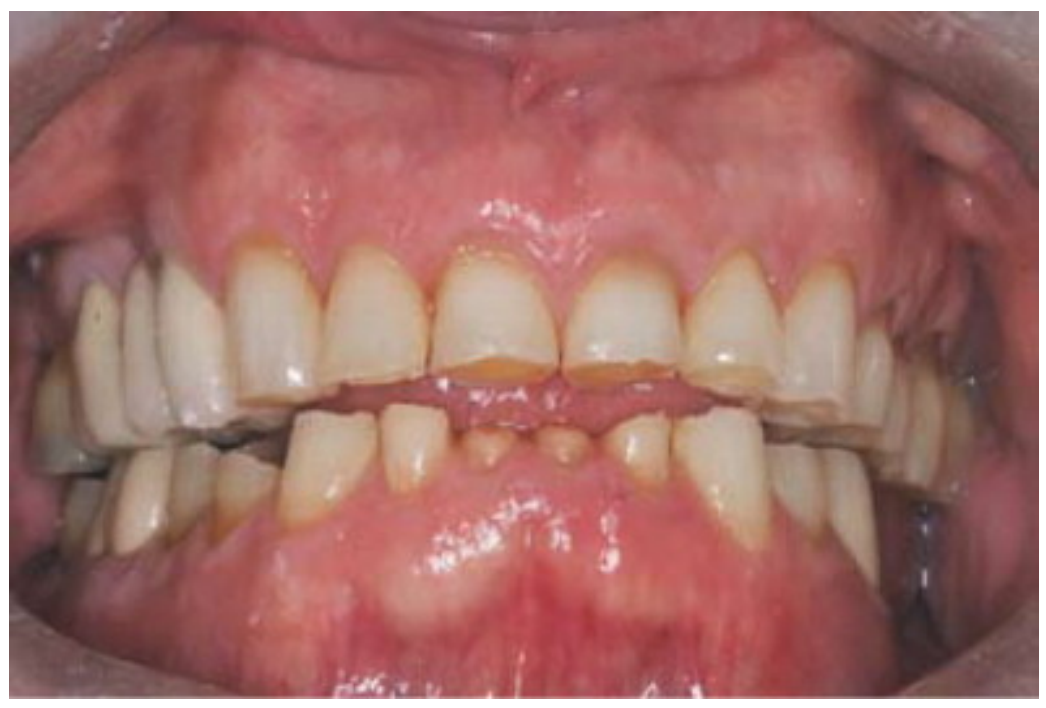

Fig. 1. Preoperative frontal view in maximum intercuspation. 


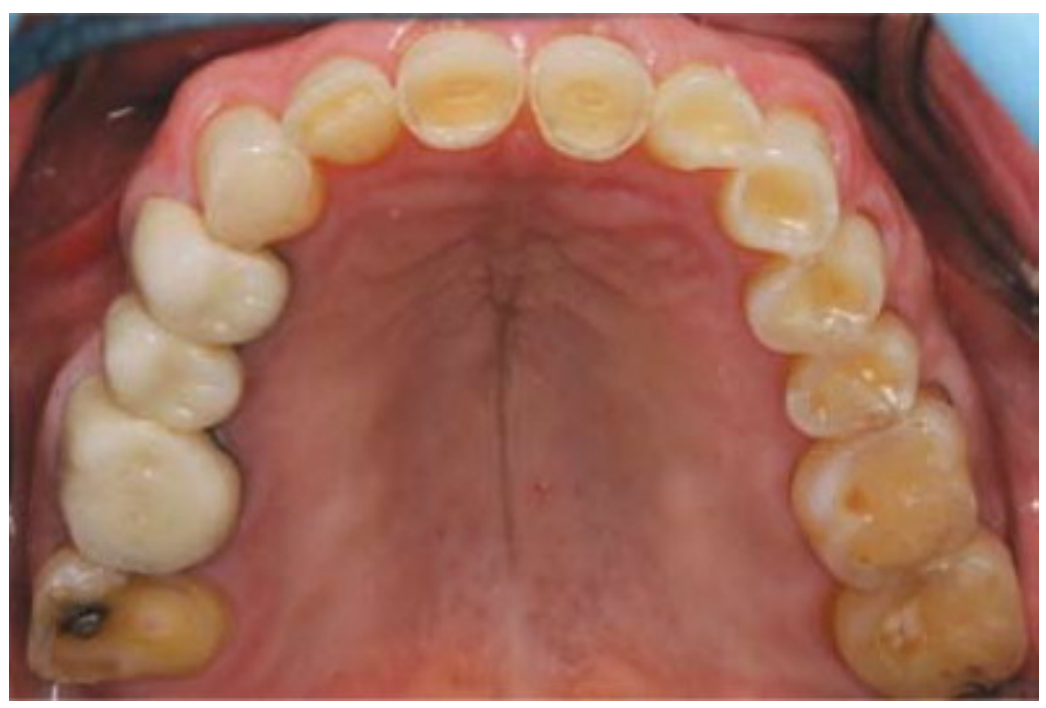

Fig. 2. Preoperative maxillary occlusal view.

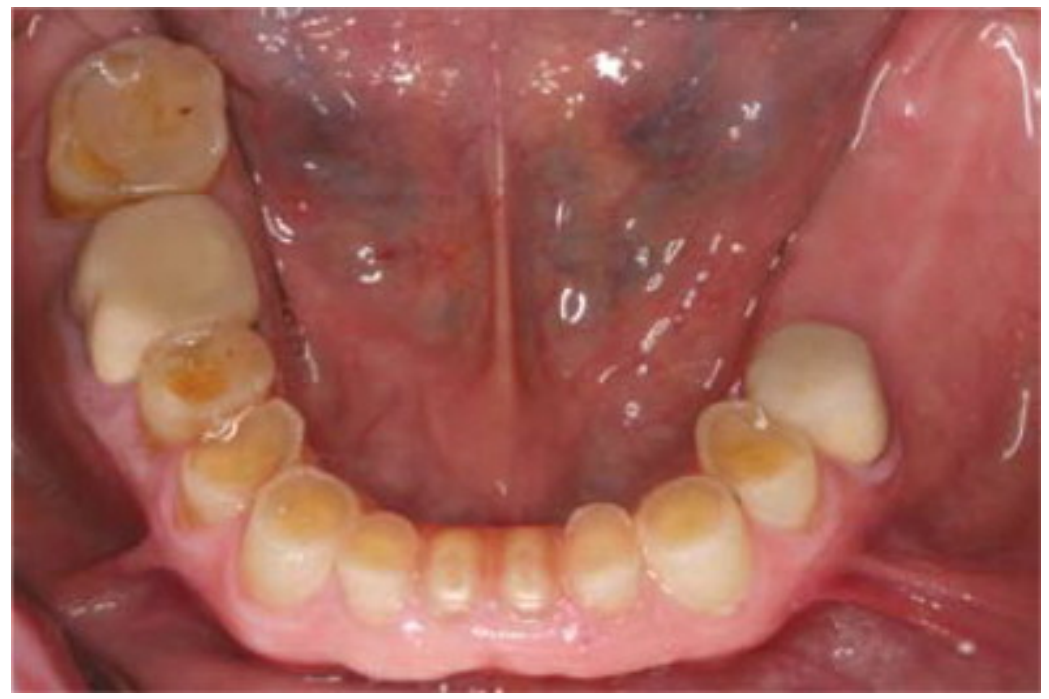

Fig. 3. Preoperative mandibular occlusal view.

Therefore, the treatment plan selected by the patient involved extraction of mandibular central incisors. Due to the amount of tooth wear, all teeth were to be restored with metal ceramic crowns. The posterior metal ceramic crowns were to be restored with metal occlusal surfaces to an increased OVD to reduce the chance of porcelain fracture. The mandibular left first and second molar edentulous spaces were to be restored with implant-supported crowns.

The diagnostic phase involved a reversible diagnostic composite resin foundation on the anterior teeth to determine the length of teeth and to evaluate phonetics and esthetics. Phonetics was assessed using the closest speaking space technique. On clinical assessment, the patient was classified as a Turner Category 2 situation demonstrating excessive tooth wear. $\underline{1}$ Although the OVD appeared to have been maintained at the clinical evaluation phase, there was insufficient tooth structure to allow for dental rehabilitation. This problem could be 
corrected by a crown lengthening procedure or by increasing the OVD. 1 Due to the age of the patient and the amount of osseous clinical crown lengthening required, it was decided to resolve the problem by attempting to increase the OVD.

To restore the patient's dentition, it was determined that the OVD would have to be increased by $3 \mathrm{~mm}$ anteriorly at the incisal guide table. Diagnostic casts were mounted in centric occlusion on a semiadjustable articulator with an adjustable medial wall (Hanau Modular Articulator; Whip Mix Corp, Louisville, Ky). A facebow transfer was made using a facebow (Hanau Spring-Bow; Whip Mix Corp) to the axis orbital plane. A maxillomandibular occlusal record was made with the help of a Lucia jig and vinyl polysiloxane occlusal registration material (Blu-Mousse; Parkell, Inc, Edgewood, NY) at the existing OVD. The pin was then dropped to $3 \mathrm{~mm}$ to achieve space for the restorative materials posteriorly. The diagnostic waxing was accomplished (Fig. 4) at this increased OVD. The BM alloy selected was Pisces Plus (Ivoclar Vivadent, Amherst, NY), a predominantly nickel-based alloy (nickel 61.5\%, chromium $22.0 \%$, aluminum $2.3 \%$ ). The patient reported no previous allergies to nickel alloys. The BM onlays were waxed on a different cast based on the diagnostic waxing, and then cast and bonded on the mandibular posterior teeth (Fig. 5) with resin cement (Panavia F2.0; Kuraray America, Houston, Tex). OVD was increased by bonding the base metal onlays to one arch only. The interocclusal space was ultimately evenly divided between the maxillary and mandibular arches at the time of definitive restoration. Direct composite resin restorations (Tetric Ceram; Ivoclar Vivadent) were bonded in the palatal aspects of the anterior teeth to the new OVD to achieve even contacts at the anterior and posterior dentition. The patient wore the $\mathrm{BM}$ onlays for a period of 2 months. During the evaluation period, the patient's anterior and posterior speaking space and function were assessed. The muscles of mastication and the temporomandibular joint were evaluated for clinical signs of discomfort. The patient was asymptomatic and comfortable during this period.

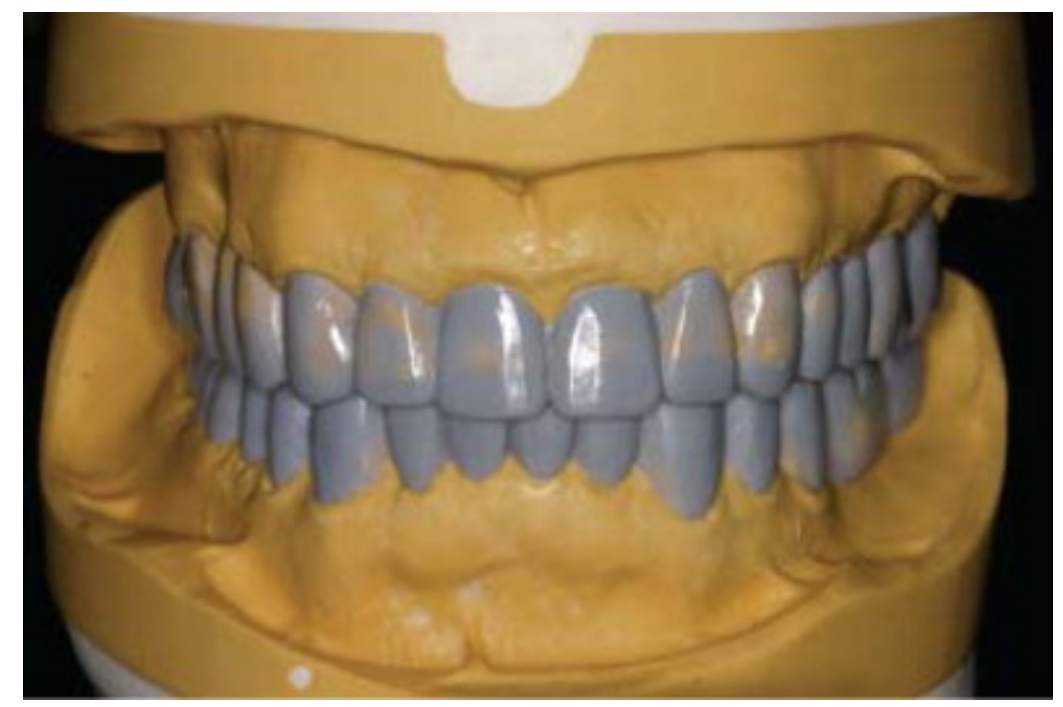

Fig. 4. Diagnostic waxing. 


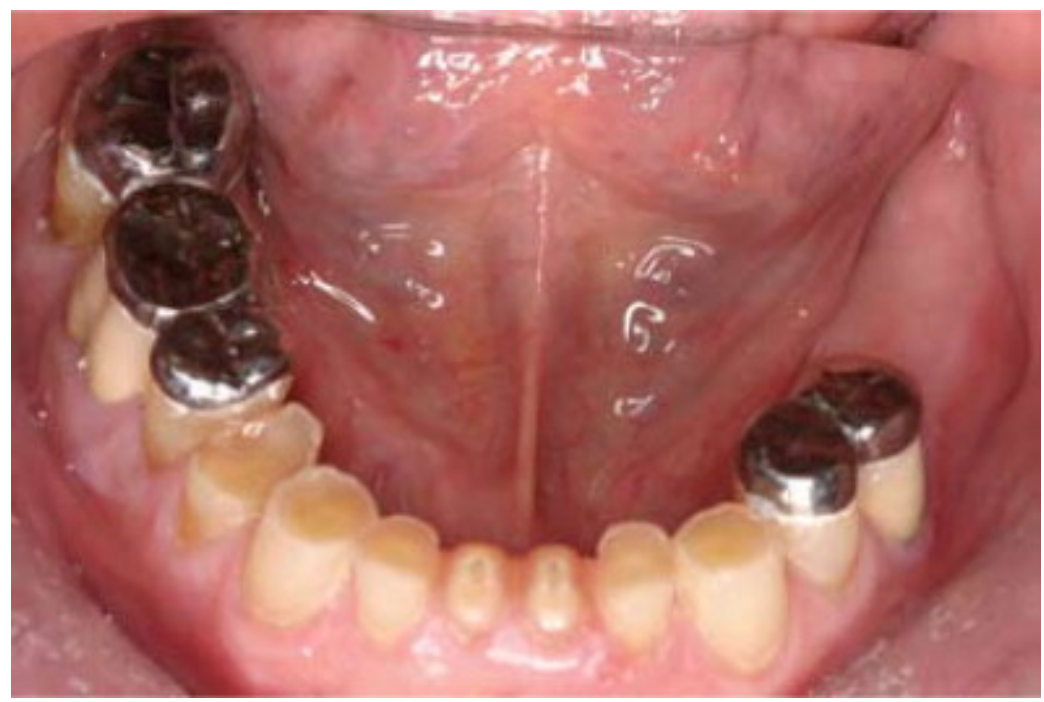

Fig. 5. Mandibular occlusal view showing bonded base metal onlays.

The evaluation phase was followed by crown preparation and the placement of provisional restorations using a segmental approach. The maxillary and mandibular anterior teeth were prepared first. Autopolymerizing acrylic resin (Jet;Lang Dental Mfg Co, Wheeling, III) provisional res torations were fabricated using a vacuum formed matrix (Copyplast; ScheuDental $\mathrm{GmbH}$, Iserlohn, Germany) produced from the diagnostic waxing and luted with provisional cement (TempBond NE; Kerr Corp, Orange, Calif) (Fig. 6). Even protrusive contacts and canine guidance were established in the provisional restorations. Esthetics and phonetics were evaluated. Definitive impressions were made with vinyl polysiloxane impression material (Affinis; Coltène/Whaledent, Inc, Cuyahoga Falls, Ohio).

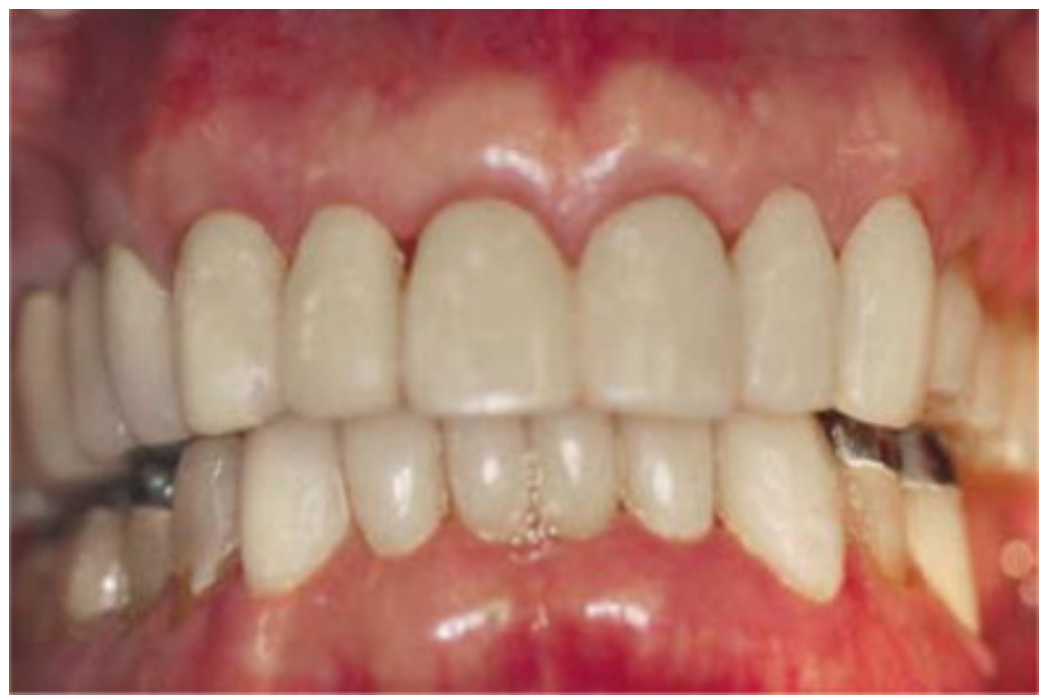

Fig. 6. Maxillary and mandibular anterior provisional restorations.

The maxillary and mandibular anterior teeth were restored with definitive restorations. Centric occlusion, even protrusive contacts, and canine guidance were established in the definitive 
anterior restoration. Arc of closure interferences were adjusted and long centric occlusion was developed in the maxillary anterior restoration to allow for freedom in anterior posterior movement. This was followed by sequential rehabilitation of the left side followed by the right side, which involved the following procedures, including: removal of the base metal onlays, crown preparation, fabrication of the provisional restoration, making of definitive impressions with vinyl polysiloxane impression material (Affinis; Coltène/Whaledent, Inc), and fabrication of definitive restorations. This was followed by trial insertion of the definitive restorations and occlusal adjustments and, finally, cementation with definitive cement (Fuji Plus; GC America, Alsip, III).

During the time the right quadrant was being definitively restored, 2 implants (NobelReplace Tapered Groovy; Nobel Biocare USA, Yorba Linda, Calif) were placed in the left posterior quadrant using a surgical guide. The implants were left to integrate for a period of 2 months with healing abutments. After completing the restoration of the right side, an implant level impression was made to restore the mandibular left first and second molar with implantsupported crowns. A temporary engaging plastic abutment (Nobel Biocare USA) was waxed and scanned with a scanner (Procera Piccolo; Nobel Biocare USA) to obtain titanium custom abutments. The definitive titanium custom abutments were evaluated intraorally and then restored with provisional crowns, which were cemented with provisional cement (TempBond NE; Kerr Corp). Then definitive impressions of the custom abutments were made for the fabrication of definitive restorations of the mandibular left first and second molars. Following the definitive cementation of all restorations (Fig. 7, Fig. 8, Fig. 9), an occlusal splint was fabricated to protect the restorations, and neutral $\mathrm{pH}$ sodium fluoride (PreviDent $1.1 \%$ BrushOn Gel; Colgate Oral Pharmaceuticals, New York, NY) was prescribed to prevent secondary decay under the restorations. The patient was advised to return every 6 months for a recall evaluation. The patient was satisfied with the improved esthetics and function of his teeth. The patient has been followed for 8 months since the completion of his treatment and remains free of any complications.

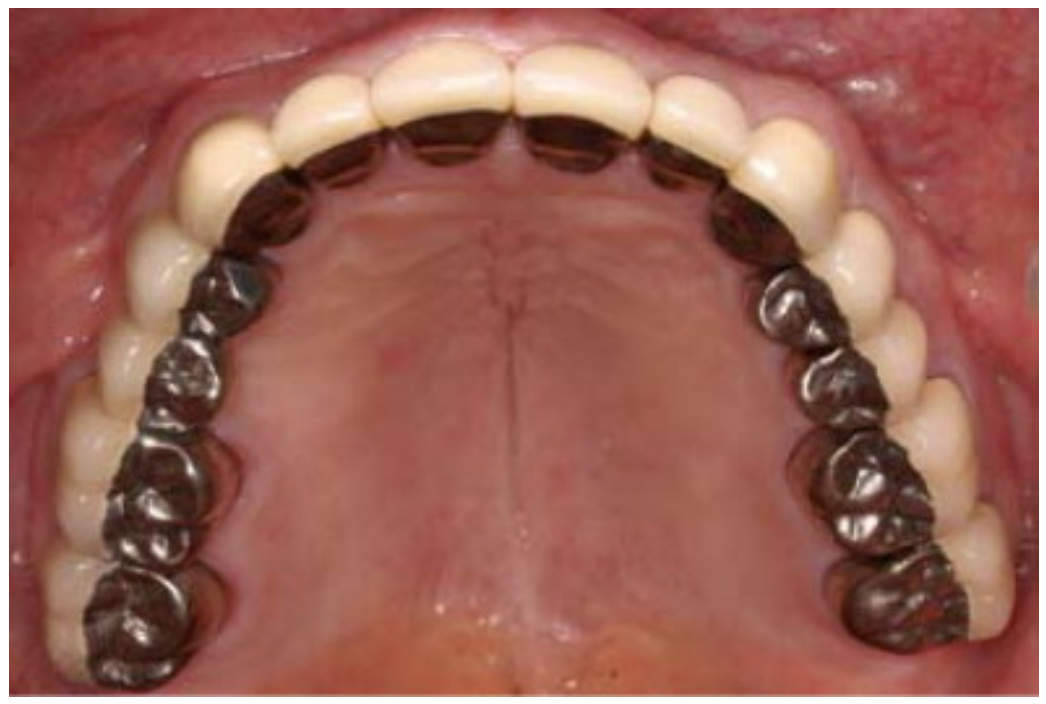

Fig. 7. Postoperative maxillary occlusal view. 


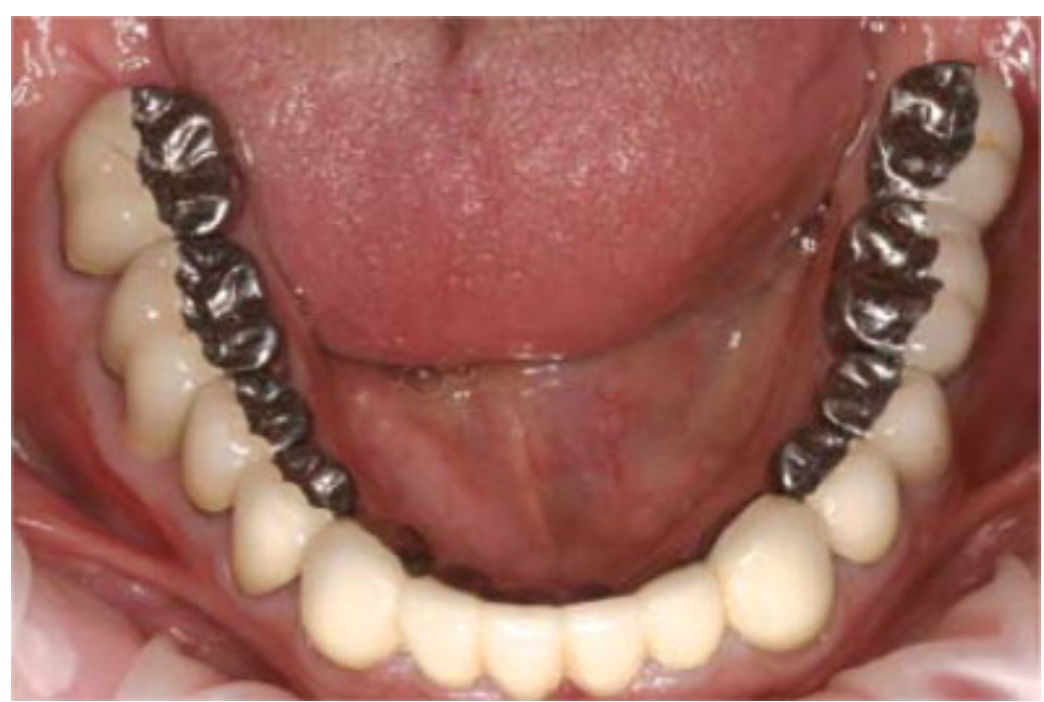

Fig. 8. Postoperative mandibular occlusal view.

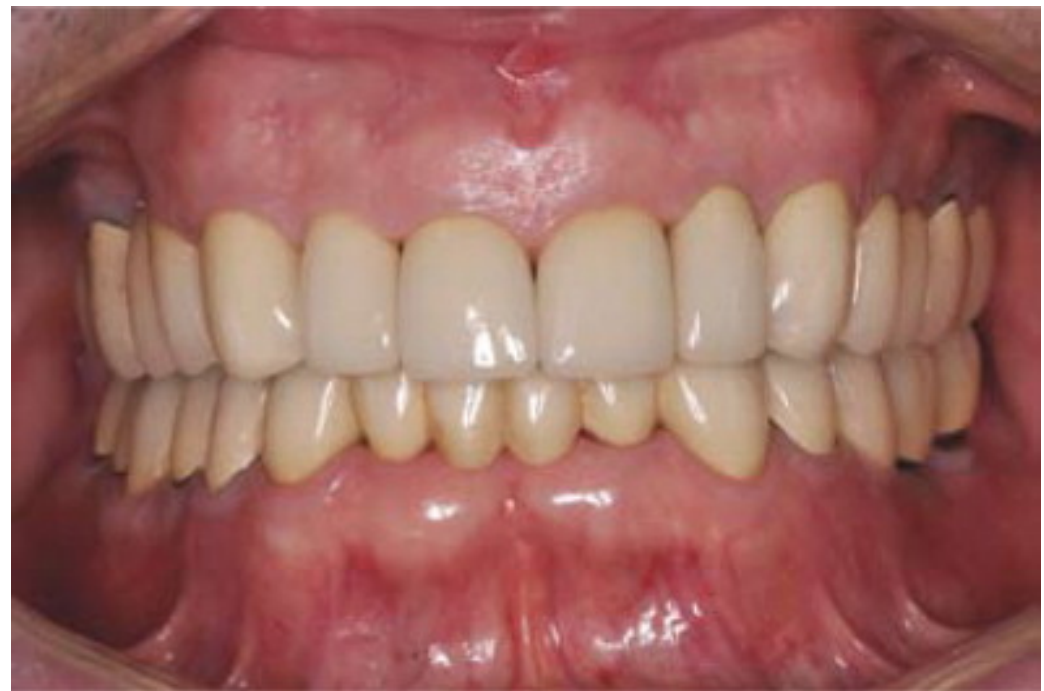

Fig. 9. Postoperative frontal view in centric occlusion.

\section{Summary}

Many situations requiring complete mouth rehabilitation present with the challenge of a lack of restorative space. This may require an increase in occlusal vertical dimension to restore the dentition to an ideal form and function. Base metal onlays can be used predictably in these situations. Due to their hardness, they can be used as a durable long-term provisional restoration, which may be a useful treatment option in patients requiring an increase in the OVD. BM onlays allow for segmental treatment, providing patient comfort and financial flexibility along with ease of treatment. These onlays offer a fairly reversible, stable, and effective method of achieving functional occlusion in situations that require complete mouth rehabilitation. 
References

${ }^{1}$ KA Turner, DM Missirlian Restoration of the extremely worn dentition J Prosthet Dent, 52 (1984), pp. 467-474

2 JO Grippo, M Simring, S Schreiner Attrition, abrasion, corrosion and abfraction revisited: a new perspective on tooth surface lesions J Am Dent Assoc, 135 (2004), pp. 11091118

${ }^{3}$ The glossary of prosthodontic terms J Prosthet Dent, 94 (2005), p. 57

${ }^{4} \mathrm{H}$ Sicher The biology of attrition Oral Surg Oral Med Oral Pathol, 6 (1953), pp. 406-412

${ }^{5}$ DC Berry, DF Poole Attrition: possible mechanisms of compensation J Oral Rehabil, 3 (1976), pp. 201-206

${ }^{6}$ C McNeill (Ed.), Science and practice of occlusion, Quintessence, Chicago (1997), pp. 429434

${ }^{7}$ A Manns, R Miralles, C Pallazi EMG, bite force and elongation of the masseter muscle under isometric voluntary contractions and variations of vertical dimension $\mathrm{J}$ Prosthet Dent, 42 (1979), pp. 674-682

${ }^{8} \mathrm{E}$ Pound The mandibular movements of speech and their seven related values $\mathrm{J}$ Prosthet Dent, 16 (1966), pp. 835-843

${ }^{9} \mathrm{ME}$ Niswonger The rest position of the mandible and centric relation J Am Dent Assoc, 21 (1934), pp. 1572-1582

10 MA Pleasure Correct vertical dimension and freeway space J Am Dent Assoc, 43 (1951), pp. $160-163$

${ }^{11}$ A Samant, JO Martin, WR Cinotti, F Moy Vertical dimension of the face and muscle tone Compendium, 7 (1986), pp. 758-759 755

12 D Sen, E Nayir, S Pamuk Comparison of the tensile bond strength of high-noble, noble, and base metal alloys bonded to enamel J Prosthet Dent, 84 (2000), pp. 561-566 